\title{
Association of atrial fibrillation susceptibility genes, atrial fibrillation phenotypes and response to catheter ablation: a gene-based analysis of GWAS data
}

Daniela Husser ${ }^{*}{ }^{\dagger}$, Petra Büttner ${ }^{\dagger}$, Laura Ueberham, Borislav Dinov, Philipp Sommer, Arash Arya, Gerhard Hindricks and Andreas Bollmann

\begin{abstract}
Background: Previous studies have suggested PITX2, KCNN3 and ZFHX3 as atrial fibrillation (AF) susceptibility genes. Single common genetic polymorphisms of those genes have been linked with AF phenotypes and rhythm outcome of AF catheter ablation although their mechanisms remain elusive. New gene-based association tests may help clarifying genotype-phenotype correlations. Therefore, we hypothesized that PITX2, KCNN3 and ZFHX3 associate with left atrial enlargement and persistent AF and subsequently with ablation outcome.
\end{abstract}

Methods and results: Samples from 660 patients with paroxysmal $(n=370)$ or persistent AF $(n=290)$ undergoing AF catheter ablation were genotyped for $\sim 1,000,000$ SNPs. Gene-based association was investigated using two different gene-based association tests (VEGAS, minSNP). Among the three candidate genes, only ZFHX3 associated with left atrial dilatation and AF recurrence after catheter ablation.

Conclusion: This study suggests a contribution of ZFHX3 to AF remodeling and response to therapy. Future and larger studies are necessary to replicate and apply these findings with an emphasis on designing AF pathophysiologybased multi-locus risk scores.

Keywords: Atrial fibrillation, Catheter ablation, Genome wide association study, Gene-based analysis

\section{Background}

Single nucleotide polymorphisms (SNPs) at chromosomes 4q25 (PITX2) [1, 2], 16q22 (ZFHX3) [3], and 1q21 (KCNN3) [4] have been shown to associate with atrial fibrillation (AF) in different genome-wide association studies (GWAS).

Catheter ablation is an established AF treatment, but arrhythmia recurrence occurs in up to $50 \%$ of patients within 1 year after ablation [5]. Previous work has consistently identified left atrial enlargement and persistent AF as clinical predictors for ablation success [6]. Recent

\footnotetext{
*Correspondence: dani11@gmx.net

${ }^{\dagger}$ Daniela Husser and Petra Büttner contributed equally to this work Department of Electrophysiology, Heart Center Leipzig, Leipzig University, Strümpellstr. 39, 04289 Leipzig, Germany
}

candidate-gene studies have linked common genetic variants with intermediate AF phenotypes such as left atrial fibrosis [7] as well as rhythm outcome after AF ablation [8-10].

However, implicated genetic variants extend over broad genomic distances, often spanning several thousands of bases raising the possibility that those loci may contain multiple independent susceptibility signals. In addition, linkage disequilibrium (LD) structure and genotyping coverage may impact on GWAS results when only analyzing single SNPs. Consequently, gene-based association tests have been introduced that are well-suited to identify genes that may increase susceptibility to complex diseases, phenotypes and response to therapy. They have been designed to detect genes that are genome-wide 
significant, but where no single SNP effect is large enough to be genome-wide significant by univariate tests.

Here, for the first time, we apply gene-based analysis of GWAS data to test the hypothesis that AF candidate genes PITX2, KCNN3 and ZFHX3 associate with left atrial enlargement and persistent $\mathrm{AF}$ and subsequently with arrhythmia recurrence following AF catheter ablation.

\section{Methods}

\section{Patients}

Six hundred-and-sixty patients with non-valvular AF undergoing de-novo radiofrequency AF catheter ablation between 2008 and 2013 were enrolled in the Leipzig Heart Center AF ablation registry. Paroxysmal AF was defined as self-terminating episodes of AF within 7 days after onset documented by ECG or an ambulatory ECG monitor. Persistent AF was defined as an AF episode either lasting longer than 7 days or requiring drug or direct current cardioversion for termination.

Transthoracic and transesophageal echocardiography (TEE) was performed prior to catheter ablation. Left atrial diameter (LAD) and left ventricular ejection fraction were determined using standard measurements and a left atrial thrombus was excluded. All class I or III antiarrhythmic medications with the exception of amiodarone were discontinued at least 5 half-lives before the procedure.

\section{AF catheter ablation and follow-up}

Left atrial catheter ablation was performed using a previously described approach $[8,11]$. In brief, patients were studied under deep propofol sedation with continuous invasive monitoring of arterial blood pressure and oxygen saturation. Non-fluoroscopic 3D catheter orientation, CT image integration, and tagging of the ablation sites were performed using Ensite NavX, Ensite Velocity (St. Jude Medical, St. Paul, MN, USA) or CARTO 3 (Biosense Webster, Diamond Bar, CA, USA). Trans-septal access and catheter navigation were performed with a steerable sheath (Agilis, St. Jude Medical, St. Paul, MN, USA). Patients presenting with AF at the beginning of the procedure were electrically cardioverted and ablation was performed during sinus rhythm (i.e. AF termination by ablation was not attempted). In all patients circumferential left atrial ablation lines were placed around the antrum of the ipsilateral pulmonary veins (irrigated tip catheter, pre-selected tip temperature of $48^{\circ} \mathrm{C}$, and maximum power of $20-40 \mathrm{~W}$ ). In patients with persistent $\mathrm{AF}$, linear lesions were added at the left atrial roof, the basal posterior wall and the left atrial isthmus or in low voltage areas (LVA) until 2011. In patients recruited between 2011 and 2013, electro-anatomical voltage mapping to characterize LVA defined as potentials below $0.5 \mathrm{mV}$ was performed as previously described [11]. After circumferential line placement, voltage and pace mapping along the ablation line were used to identify and close gaps. The isolation of all pulmonary veins with bidirectional block was verified with a multipolar circular mapping catheter and was defined as the procedural endpoint. Burst pacing from coronary sinus (down to $200 \mathrm{~ms}$ ) was performed at the end of the procedure. If sustained AF was induced, patients were electrically cardioverted and no additional ablation was performed. If atrial tachycardia was induced, those were mapped and ablated.

After ablation, class I and III antiarrhythmic drugs were not reinitiated. Oral anticoagulation was prescribed for 6 months, and proton pump inhibitors were added for 4 weeks. All patients were followed in the outpatient clinic for 12 months after the ablation. During this follow-up period, 7-day Holter ECG recordings were performed 3, 6 and 12 months after the ablation. Additional ECGs and Holter ECG recordings were obtained when patients' symptoms were suggestive of AF. AF recurrence was defined as a documented AF episode lasting longer than $30 \mathrm{~s}$ between 3 and 12 months after the ablation (thus, including a 3-month "blanking period"). All patients with sustained early recurring AF underwent direct cardioversion. Additional drug administration was left to the discretion of the treating physician.

\section{Sample processing}

Blood samples were obtained in EDTA test tubes in fasting state prior ablation. Genomic DNA was isolated using a commercial kit according to the manufacturer's recommendations (PeqLab, Erlangen, Germany). Genotyping was performed using HumanOmniExpressExome-8-v1.2 arrays comprising about one million single nucleotide polymorphisms (SNPs) according to established protocols (Illumina, San Diego, US).

\section{Data analysis and statistics}

Raw data was compiled using GenomeStudio (Illumina) software and exported to PLINK GWAs analysis package [12]. Using PLINK tool set the data was tested for consistency. Samples with a call rate $<95 \%$ were excluded. Single SNPs had to meet the following criteria: minor allele frequencies $(\mathrm{MAF})>0.01$, call rate $>95 \%$, Hardy-Weinberg equilibrium (HWE) significance threshold $>0.0001$. Otherwise they were excluded from further analysis.

Association of genotypes with LAD was detected using linear regression with adjustment for age, gender, body mass index and AF type. Association of genotypes with AF type (persistent AF) and arrhythmia recurrence was detected using logistic regression analysis with adjustment for age and gender. 
Illumina's exome arrays contain specific "exm-SNPs" which were assigned to their corresponding dbSNP rs IDs prior further analysis.

We used two different gene-based tests (minSNP, VEGAS [versatile gene-based association study]) whose performance depend on different genetic architectures (multiple independent signals versus single signal in a gene, size of gene, patterns of LD in the gene and so on) [13]. minSNP calculates a gene-based $P$-value either directly from a parametric distribution or by using the permuted $P$-value of the best individual SNP association within the given gene. minSNP computes single-SNP F-statistics for each SNP within a gene and uses the best F-statistic within that gene as its test statistic, which is then converted to a $P$-value with gene-based permutations to correct for gene size [13]. VEGAS uses the sum of $X^{2}$ for an individual SNP to generate a test statistic suggested for the gene. The $P$-value of the gene is then computed after accounting for LD and the number of SNPs in each gene [14].

SNPs within $200 \mathrm{kB}$ of the candidate genes were selected for inclusion. This range was chosen based on previous studies implicating common 4q25 variants $195 \mathrm{kB}$ upstream of PITX2 in AF susceptibility and response to therapy.

We applied a two-stage analysis plan. First, we identified consistently associated genes with LAD and AF type. For this step, a Bonferroni correction was applied to account for the analysis of three genes and two phenotypes ( $P$-value less than $0.05 / 6=0.0083$ ).

Second, association of those identified gene(s) with AF ablation outcome was tested. For this analysis, we divided the cohort into two data sets based on the ablation period and strategy (data set $1: \mathrm{n}=496,2008-2011$, pulmonary vein isolation \pm linear lesions; data set $2: \mathrm{n}=164$, 2011-2013, pulmonary vein isolation \pm voltage-guided substrate modification) and performed a meta-analysis on individual data for the entire cohort.

eQTL analyses of significant SNP(s) were performed using the publicly available genotype-tissue expression portal (GTEx) of the Broad Institute of Harvard and MIT (GTex, Broad Institute, Boston, MA, USA; http://www. gtexportal.org/home/).
Clinical variables are presented as mean \pm one standard deviation or percentages. They were compared between patients with and without AF recurrence using Chi square or Student's t-test.

\section{Results}

\section{Patient characteristics}

The study population included 660 patients with a history of paroxysmal $(n=370)$ or persistent AF $(n=290$, Table 1). LAD was available in 538 of those data sets and could not be analyzed due to insufficient echo conditions or performance of TEE only in the remaining patients. LAD was significantly larger in patients with persistent AF ( $41 \pm 5$ vs. $45 \pm 6 \mathrm{~mm}, P<1.0 \mathrm{E}-3)$ compared to patients with paroxysmal AF. Both data sets were different with respect to gender, AF type, LAD and LVEF but AF recurrence rate was identical (Table 1).

AF recurrence between 3 and 12 months was observed in $48 \%$ (Table 2) and was associated with LAD (OR 1.05 per $\mathrm{mm}$ increase, 95\% CI 1.02-1.08, $P=1.0 \mathrm{E}-3$ ) and persistent AF (OR 2.1; 95\% CI 1.567-2.931, $P=2.0 \mathrm{E}-6$ ) in multivariate analysis.

Genotyping call rate in all subjects was $>95 \%$ except in three samples $(<85 \%)$ that were excluded from further analysis.

\section{Gene-based associations}

Results from the gene-based tests for association between AF candidate genes and AF phenotypes are summarized in Table 3. KCNN3 associated with LAD and AF type but failed to reach pre-defined significance threshold while PITX2 was not consistently associated with any of the phenotypes. ZFHX3 was significantly associated with LAD in both tools and AF type in VEGAS but not in minSNP.

Therefore, association between $Z F H X 3$ and AF recurrence was assessed (Fig. 1). ZFHX3 associated with AF recurrence in both data sets and subsequently in the entire cohort $(P=0.001$ in VEGAS and $P=0.0288$ in $\operatorname{minSNP}$ ).

By accessing the publicly available GTEx, a significant association between the top ZFHX3 SNP (Fig. 2), rs12373097 (OR for LRAF 1.57, 95\% CI 1.18-2.08,

Table 1 Patient characteristics

\begin{tabular}{lllll}
\hline & Total $(\mathbf{n = 6 6 0 )}$ & Data set $\mathbf{1}(\mathbf{n}=\mathbf{4 9 6})$ & Data set $\mathbf{2}(\mathbf{n}=\mathbf{1 6 4})$ & $\boldsymbol{P}$-value \\
\hline Age (years) & $60 \pm 10$ & $59 \pm 10$ & $61 \pm 10$ & $\mathrm{~ns}$ \\
Male gender (\%) & 68 & 66 & 75 & $2.1 \mathrm{E}-02$ \\
Body mass index $\left(\mathrm{kg} / \mathrm{m}^{2}\right)$ & $28.9 \pm 4.6$ & $28.9 \pm 4.5$ & $29.0 \pm 4.7$ & $\mathrm{~ns}$ \\
Idiopathic AF (\%) & 14 & 13 & 15 & $\mathrm{~ns}$ \\
Persistent AF (\%) & 44 & 38 & 61 & $3.3 \mathrm{E}-07$ \\
LAD (mm) & $43 \pm 6$ & $42 \pm 6$ & $44 \pm 6$ & $2.0 \mathrm{E}-03$ \\
LVEF (\%) & $59 \pm 10$ & $60 \pm 9$ & $57 \pm 10$ & $1.0 \mathrm{E}-03$ \\
\hline
\end{tabular}


Table 2 Patient characteristics in patients with and without AF recurrence

\begin{tabular}{lll}
\hline & AF recurrence $(\mathbf{n}=\mathbf{3 1 8})$ & No recurrence $(\mathbf{n}=\mathbf{3 4 1})$ \\
\hline Age (years) & $61 \pm 10$ & $59 \pm 10$ \\
Male gender $(\%)$ & 70 & 67 \\
Body mass index $\left(\mathrm{kg} / \mathrm{m}^{2}\right)$ & $29.2 \pm 4.6$ & $28.7 \pm 4.5$ \\
Persistent AF (\%) & 53 & 35 \\
LAD (mm) & $44 \pm 6$ & $42 \pm 6$ \\
LVEF (\%) & $58 \pm 10$ & $59 \pm 9$ \\
\hline
\end{tabular}

One patient was lost to follow-up

Table 3 Gene-based association results

\begin{tabular}{|c|c|c|c|c|}
\hline & \multicolumn{2}{|l|}{ LAD } & \multicolumn{2}{|l|}{ AF type } \\
\hline & VEGAS & $\operatorname{minSNP}$ & VEGAS & $\operatorname{minSNP}$ \\
\hline KCNN3 & 0.0153 & 0.0396 & $<0.00001$ & 0.0348 \\
\hline PITX2 & 0.0083 & ns & ns & ns \\
\hline ZFHX3 & 0.0001 & 0.0043 & 0.0001 & $\mathrm{~ns}$ \\
\hline
\end{tabular}

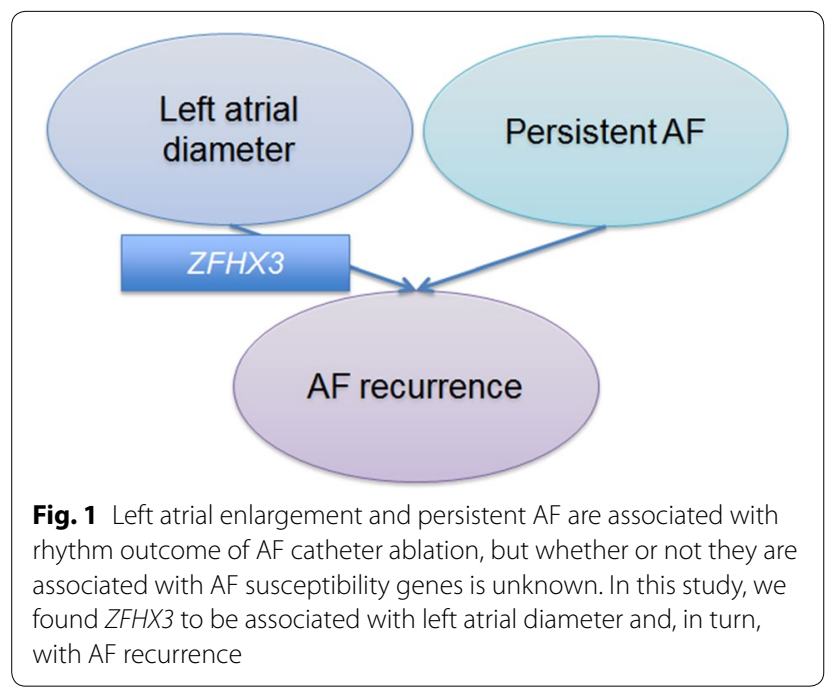

$P=0.002)$, with a higher expression of ZFHX3 in fibroblasts $(P=8.0 \mathrm{E}-8)$ was found. This SNP had a minor allele frequency of 20.3 with $32 \%$ of patients being heterozygous and $4 \%$ being homozygous.

\section{Discussion}

\section{Main findings}

This study is the first to explore the association of AF candidate genes and AF remodeling-associated phenotypes such as left atrial enlargement and AF type as well as response to $\mathrm{AF}$ catheter ablation using gene-based analysis of GWAS data. ZFHX3 was associated with left atrial diameter and ablation outcome in two different gene-based tests.

\section{Association between genotype, AF phenotypes and AF ablation outcomes}

Different AF susceptibility alleles have been associated with non-pulmonary vein triggers and left atrial scar [7]. Interestingly, of those SNPs, one ZFHX3 SNP (rs7193343) was the only genetic variant that contributed to increased risk of both phenotypes. Somewhat conflicting results have been obtained with left atrial parameters. There were no associations between candidate SNPs and several left atrial measures in the Framingham cohort. However, after excluding prevalent AF, a single SNP (rs2200733) in proximity to PITX2 was associated with left atrial volume following adjustment for body surface area. A second SNP (rs2106261) in proximity to the $Z F H X 3$ gene was related to $\mathrm{LAD}$ [15].

Common genetic variants have also been associated with ablation outcome. Of those, the $4 \mathrm{q} 25$ variants have been shown the strongest association in single-center studies and a recent meta-analysis, $[8,10]$ although results have not been consistent $[16,17]$.

In this study, we focused on gene-based tests and first identified an association between genotype and AF phenotypes LAD and AF type and then between genotype and AF ablation outcome. This was based on the fact that left atrial enlargement and AF persistence have consistently been linked with response to AF ablation outcome [6]. Our study revealed associations of the ZFHX3 gene with left atrial dilatation and subsequently with rhythm outcome. This finding is supported by one previous study that identified the rs2106216 ZFHX3 genetic polymorphism as independent predictor of a good ablation response [15] and strong biological evidence linking $Z F H X 3$ with $\mathrm{AF}$ and cardiac remodeling discussed below.

\section{ZFHX3 and AF}

$Z F H X 3$ encodes a cardiac transcription factor containing multiple homeodomains and zinc finger motifs and common $Z F H X 3$ variants have been shown to increase AF risk [3, 18]. Although their cardiac mechanisms 


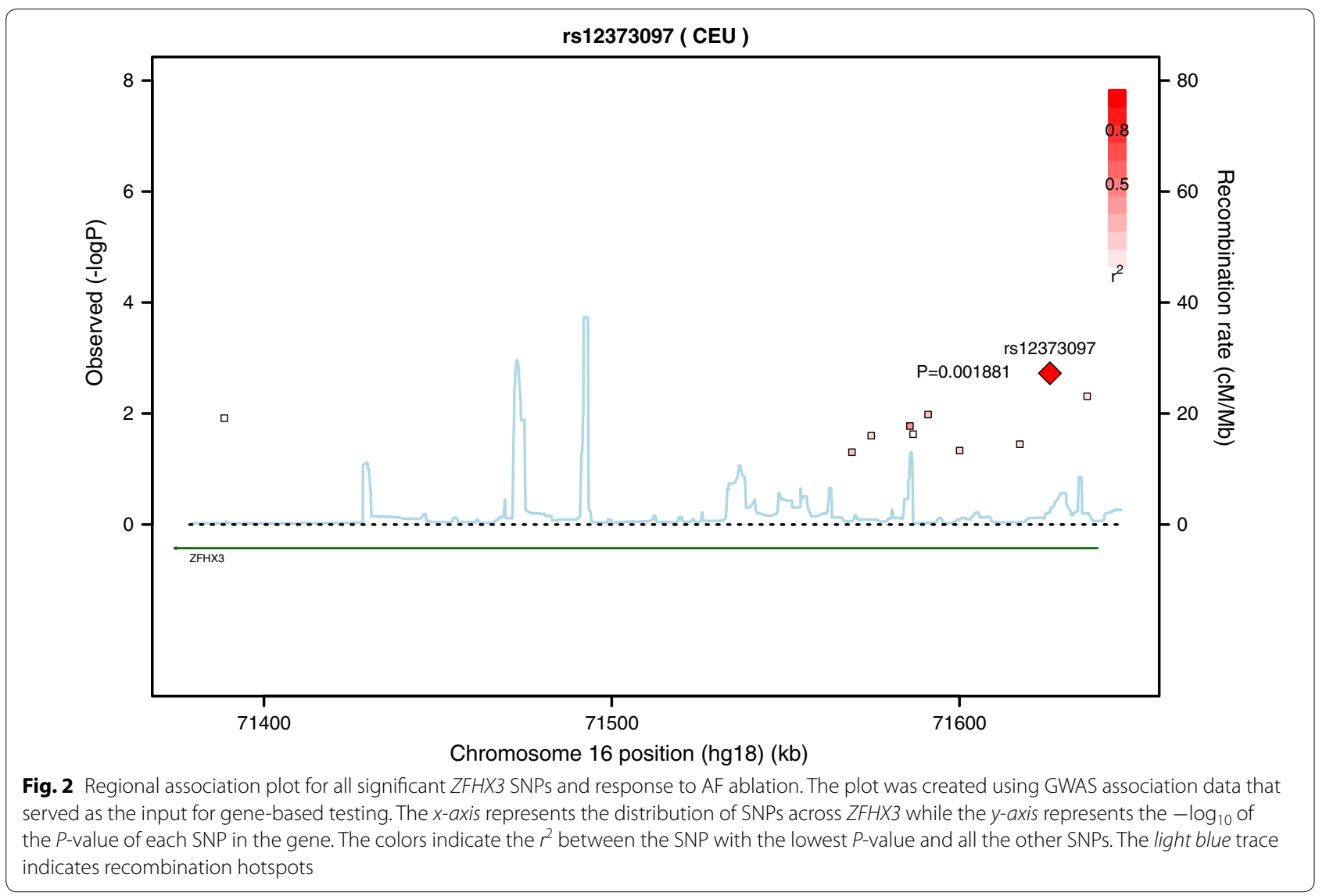

underlying AF remain elusive, several gene effects have been demonstrated. For instance, ZFHX3 is related to JAK/STAT signaling cascade that mediates the inflammatory process thereby contributing to electrical and structural remodeling of the atrium with inflammatory changes [19]. Interestingly, cross-regulation of gene expression of AF candidate genes has been suggested as molecular basis for gene-gene interactions [20]. The most significant ZFHX3 SNP in our study, rs12373097, has been found by eQTL analysis to predict higher expression of $Z F H X 3$. ZFHX3 positively regulates expression of PITX2 and both ZFHX3 and PITX2 positively regulate expression of NPPA. Atrial natriuretic peptide, the NPPA product, in turn, plays a key role in cardiac electrophysiology through APD shortening and calcium influx reduction, modulating the autonomic nervous system and regulating the function of cardiac ion channels [21]. In addition, both ZFHX3 and PITX2 impact on other downstream genes such as $T B X 5$, $N K X 2.5, K C N Q 1$ and $S C N 1 B$ that play critical roles in cardiac electrophysiology and AF [20]. Of those, TBX5 modulation has very recently been found to profoundly alter cardiac channel gene expression and to cause primary, spontaneous AF in mice [22].
Taken together, those and our findings implicate ZFHX3 in several AF-remodeling associated processes that may underlie left atrial dilatation and AF progression and subsequently response to AF catheter ablation.

\section{Limitations}

Our study is based on small sample size. However, it was based on the three strongest candidate genes that were selected for gene-based analysis of GWAS data a priori. In other words, although we used GWAS data, we applied a hypothesis-driven approach. Furthermore, we addressed this by using well-defined intermediate AF phenotypes that are known interrelated markers of AF ablation outcome. We only selected the gene that showed associations with intermediate AF phenotypes for further analysis of its association with ablation outcome. Finally, we required the genotype-phenotype correlation to be present in two different gene-based analysis tools accounting for different genetic architectures (multiple independent signals versus single signal in a gene, size of gene, patterns of LD).

Although we have used two data sets reflecting changes in patient characteristics and ablation strategies for ablation outcome, they came from the same institution with 
all inherent limitations for genetic studies but advantage of coherent patient selection, operators and followup. Despite those differences in clinical characteristics ablation outcome was identical. Nevertheless, ablation approaches in persistent AF are evolving and linear lesion sets have lessened in popularity due to no incremental benefit and even pro-arrhythmia. This may impact on type of arrhythmia recurrence and should be considered when assessing recurrence rates and comparing this to other studies.

This study does not contribute to the elaboration of molecular mechanisms which was beyond the scope of this study and did not address the question whether or not genotype impacts on proarrhythmic ablation effects or AF progression.

Finally, ablation outcome was assessed with serial clinical and prolonged Holter ECG monitoring which is in line with current recommendations but can nevertheless miss common asymptomatic AF episodes [23].

\section{Conclusions}

These findings suggest a contribution of $Z F H X 3$ to $\mathrm{AF}$ remodeling and response to AF catheter ablation. Future and larger studies are necessary to replicate and apply these findings with a clinical emphasis on designing AF pathophysiology-based multi-locus risk scores.

\section{Abbreviations}

AF: atrial fibrillation; GWAS: genome-wide association study/studies; LAD: left atrial diameter; SNP: single nucleotide polymorphism.

\section{Authors' contributions}

$\mathrm{DH}, \mathrm{PB}$ and $\mathrm{AB}$ conceived and initiated the project, analyzed the data and wrote the manuscript. $L U, B D, P S, A A, G H$ and $A B$ collected the data. $L U, P B$ did the laboratory work. LU and PS made manuscript revisions. All authors reviewed, contributed and approved the final manuscript.

\section{Acknowledgements}

None.

\section{Competing interests}

The authors declare that they have no competing interests.

\section{Availability of data and materials}

The datasets used and analyzed during the current study are available from the corresponding author on reasonable request.

\section{Consent for publication}

This manuscript contains no individual person's data.

\section{Ethics approval and consent to participate}

The study protocol was approved by the Ethics Committee of the Leipzig University Medical Faculty and performed in accordance with the 1964 Helsinki declaration and its later amendments. Informed consent was obtained from all individual participants included in the study.

\section{Funding}

This study was supported by the Volkswagen Foundation Germany through the Lichtenberg professorship program to DH (\# 84901). This study was supported in part by the Heart Center Leipzig.
Received: 14 November 2016 Accepted: 23 March 2017

Published online: 05 April 2017

References

1. Gudbjartsson DF, Holm H, Gretarsdottir $\mathrm{S}$, et al. A sequence variant in ZFHX3 on 16q22 associates with atrial fibrillation and ischemic stroke. Nat Genet. 2009;41:876-8.

2. Lubitz $S A$, Lunetta $K L$, Lin $H$, et al. Novel genetic markers associate with atrial fibrillation risk in Europeans and Japanese. J Am Coll Cardiol. 2014;63:1200-10.

3. Gudbjartsson DF, Arnar DO, Helgadottir A, et al. Variants conferring risk of atrial fibrillation on chromosome 4q25. Nature. 2007;448:353-7.

4. Ellinor PT, Lunetta KL, Glazer NL, et al. Common variants in KCNN3 are associated with lone atrial fibrillation. Nat Genet. 2010;42:240-4.

5. Ganesan AN, Shipp NJ, Brooks AG, et al. Long-term outcomes of catheter ablation of atrial fibrillation: a systematic review and meta-analysis. J Am Heart Assoc. 2013:2:e004549.

6. D'Ascenzo F, Corleto A, Biondi-Zoccai G, et al. Which are the most reliable predictors of recurrence of atrial fibrillation after transcatheter ablation?: a meta-analysis. Int J Cardiol. 2013;167:1984-9.

7. Mohanty S, Hall AW, Mohanty P, et al. Novel association of polymorphic genetic variants with predictors of outcome of catheter ablation in atrial fibrillation: new directions from a prospective study (DECAF). J Interv Card Electrophysiol. 2016:45:7-17.

8. Husser D, Adams V, Piorkowski C, Hindricks G, Bollmann A. Chromosome $4 \mathrm{q} 25$ variants and atrial fibrillation recurrence after catheter ablation. J Am Coll Cardiol. 2010;55:747-53.

9. Park J, Lee J, Yang P, et al. Good responders to catheter ablation for longstanding persistent atrial fibrillation: clinical and genetic characteristics. J Cardiol. 2016. doi:10.1016/j.jjcc.2016.04.017.

10. Shoemaker MB, Bollmann A, Lubitz SA, et al. Common genetic variants and response to atrial fibrillation ablation. Circ Arrhythm Electrophysiol. 2015:8:296-302.

11. Dinov B, Kosiuk J, Kircher S, et al. Impact of metabolic syndrome on left atrial electroanatomical remodeling and outcomes after radiofrequency ablation of nonvalvular atrial fibrillation. Circ Arrhythm Electrophysiol. 2014;7:483-9.

12. Purcell S, Neale B, Todd-Brown K, et al. PLINK: a tool set for wholegenome association and population-based linkage analyses. Am J Hum Genet. 2007:81:559-75

13. Huang H, Chanda P, Alonso A, Bader JS, Arking DE. Gene-based tests of association. PLoS Genet. 2011;7:e1002177.

14. Liu JZ, McRae AF, Nyholt DR, et al. A versatile gene-based test for genome-wide association studies. Am J Hum Genet. 2010;87:139-45.

15. Magnani JW, Yin X, McManus DD, et al. Genetic loci associated with atrial fibrillation: relation to left atrial structure in the Framingham Heart Study. J Am Heart Assoc. 2014;3:e000616.

16. Choi E, Park JH, Lee J, et al. Korean atrial fibrillation (AF) network: genetic variants for AF do not predict ablation success. J Am Heart Assoc. 2015;4:e002046.

17. Kiliszek M, Kozluk E, Franaszczyk M, et al. The 4q25, 1q21, and 16q22 polymorphisms and recurrence of atrial fibrillation after pulmonary vein isolation. Arch Med Sci. 2016;12:38-44.

18. Benjamin EJ, Rice KM, Arking DE, et al. Variants in ZFHX3 are associated with atrial fibrillation in individuals of European ancestry. Nat Genet. 2009:41:879-81.

19. Jiang Q, Ni B, Shi J, et al. Down-regulation of ATBF1 activates STAT3 signaling via PIAS3 in pacing-induced HL-1 atrial myocytes. Biochem Biophys Res Commun. 2014;449:278-83.

20. Huang $Y$, Wang $C$, Yao Y, et al. Molecular basis of gene-gene interaction: cyclic cross-regulation of gene expression and post-GWAS gene-gene interaction involved in atrial fibrillation. PLoS Genet. 2015;11:e1005393.

21. Perrin MJ, Gollob MH. The role of atrial natriuretic peptide in modulating cardiac electrophysiology. Heart Rhythm. 2012;9:610-5.

22. Nadadur RD, Broman MT, Boukens B, et al. Pitx2 modulates a Tbx5dependent gene regulatory network to maintain atrial rhythm. Sci Transl Med. 2016:8:354.

23. Verma A, Champagne J, Sapp J, et al. Discerning the incidence of symptomatic and asymptomatic episodes of atrial fibrillation before and after catheter ablation (DISCERN AF): a prospective, multicenter study. JAMA Intern Med. 2013;173:149-56. 